\title{
Beneficial effects of prolonged blood pressure control after carotid artery stenting
}

This article was published in the following Dove Press journal:

Clinical Interventions in Aging

6 January 2017

Number of times this article has been viewed

\author{
Anna Chang' \\ Huei-Fong Hung ${ }^{2}$ \\ Fang-I Hsieh ${ }^{3}$ \\ Wei-Hung Chen ${ }^{1,4}$ \\ Hsu-Ling Yeh ${ }^{1,5}$ \\ Jiann-Horng Yeh ${ }^{1,6}$ \\ Hou-Chang Chiu ${ }^{1,6}$ \\ Li-Ming Lien ${ }^{1,4}$
}

'Department of Neurology, Shin Kong Wu Ho-Su Memorial Hospital, Taipei, ${ }^{2}$ Department of Cardiology, Shin Kong Wu Ho-Su Memorial Hospital, Taipei, ${ }^{3}$ School of Public Health, College of Public Health, Taipei Medical University, Taipei, ${ }^{4} \mathrm{School}$ of Medicine, College of Medicine, Taipei Medical University, Taipei, ${ }^{5}$ nstitute of Public Health, National Yang-Ming University, Taipei, ${ }^{6}$ School of Medicine, College of Medicine, Fu Jen Catholic University, Taipei, Taiwan
Correspondence: Li-Ming Lien Department of Neurology, Shin Kong Wu Ho-Su Memorial Hospital, 95, Wen Chang Road, Shih Lin, Taipei III, Taiwan

Tel +8862 283322 I I ext 207 I

Email limingmd@ms28.hinet.net
Objectives: The main purpose of this study was to investigate whether carotid artery stenting (CAS) plus medicine in patients with severe carotid artery stenosis provide a better long-term blood pressure (BP) control compared to other medical treatments alone. The other aim was to explore the correlation between post-CAS hypotension within $6 \mathrm{~h}$ and long-term BP reductions after CAS.

Materials and methods: Patients with severe carotid stenosis were recruited either in the CAS group or in the medication group. BPs and the number of classes of antihypertensive agents were recorded at baseline, 6, and 12 months. Extra BP information was collected at 6 h, 3 days, and 1 month after CAS. Univariate and multivariate linear regressions were performed to test the relationship of BP changes among CAS and medication groups after 6 and 12 months of follow-up. Univariate linear regressions were also used to determine the correlations between the mean or maximal systolic BP (SBP) reductions at $6 \mathrm{~h}$ and 1 year post-CAS.

Results: In total, 72 members in the CAS group and 82 members in the medication group were recruited. Compared with the medication group, patients in the CAS group had greater BP reductions at 6 and 12 months of follow-up after adjusting for confounding factors $(13.56 \mathrm{mmHg}$ at 6 months, $P=0.0002 ; 16.98 \mathrm{mmHg}$ at 12 months, $P<0.0001$ ). This study also shows significant positive correlations between the mean or maximal SBP reductions $6 \mathrm{~h}$ post-CAS and SBP reductions 1 year post-CAS ( $\beta=0.20 \pm 0.07, P=0.0067$ and $\beta=0.47 \pm 0.10, P<0.0001$, respectively). Conclusion: As compared to medical treatment alone, CAS may provide significant beneficial effect on long-term BP control 1 year post-CAS. Furthermore, SBP reductions $6 \mathrm{~h}$ post-CAS may predict the SBP reductions 1 year post-CAS.

Keywords: carotid artery stenosis, hemodynamics, cerebral autoregulation, baroreflex, poststenting hypotension, antihypertensive agents

\section{Introduction}

Hemodynamic hypotension is a well-recognized phenomenon observed in patients shortly after undergoing carotid artery stenting (CAS); its incidence rate ranges from $10 \%$ to $42 \%$. ${ }^{1}$ However, there has been conflicting results on whether this hypotensive effect of CAS sustains for a long term. Altinbas et al reported no significant blood pressure (BP) reduction 1 year post-CAS, although less number of patients were required to receive antihypertensive agents. ${ }^{2}$ Nevertheless, Chung et al provided supportive evidence and showed BP reductions 1 year post-CAS in two other similar studies. ${ }^{3,4}$ Although a previous study has established independent risk factors of short-term post-CAS hypotension, including older age and female sex, ${ }^{5}$ no study has attempted to identify a link between hemodynamic changes in the short and long term in patients undergoing CAS.

On the basis of the fact that those patients who undergo CAS may have improved cerebral autoregulation in long term, this study has hypothesized that BP control 
would improve after CAS and that medical treatment alone would sustain BP long term by either decreasing BP levels or diminishing the amount of antihypertensive agents required for optimized BP control. In addition, this study has sought to assess the predictive value of each validated definitions of post-CAS hypotension within $6 \mathrm{~h}$ to identify patients prone to significant long-term BP reductions.

\section{Materials and methods}

\section{Patients}

This study conducted a retrospective cohort study of all patients with severe internal carotid artery (ICA) stenosis between March 2005 and March 2013 at the Shin Kong Wu Ho-Su Memorial Hospital, a tertiary-centered hospital in northern Taiwan. All patients had severe ICA stenosis with $>70 \%$ of diameter, which was defined as peak systolic velocity $\geq 200 \mathrm{~cm} / \mathrm{s}$ at extracranial carotid Doppler evaluation, and without occlusion. ${ }^{6}$ Patients in the CAS group included those who chose to undergo CAS and receive medication, whereas patients in the medication group received only the medication. Clinical data were extracted from the medical file of each patient. Patients were excluded from the study if they had any life-threatening or disabling conditions that could interfere with the interpretation of outcomes (eg, shock, unstable coronary artery disease or heart failure, recurrent cerebral infarcts, and death) in the past year, or a history of manipulation of the other side of the carotid artery in the past year. Patients with missing BP or antihypertensive agent data were also excluded from the analysis. CAS was performed using standard procedures, including placement of a protection device, pre-stent angioplasty, stent placement with a self-expandable stent, and post-stent angioplasty if necessary. The protocols and informed consents were approved by the Institutional Review Board of Shin Kong Wu Ho-Su Memorial Hospital, and all participants gave written informed consent for using their medical files in this study.

\section{Treatment and follow-up}

Systolic BP (SBP) and diastolic BP (DBP) were recorded for all patients in the CAS group at baseline using the mean $\mathrm{BP}$ records of the day of admission and the night before and morning of CAS. In addition, SBP and DBP were recorded 6 h, 3 days, 1 month, 6 months, and 12 months post-CAS. SBP and DBP were recorded at baseline in the medication group using the mean of three consecutive BP measurements at an outpatient clinic before the carotid Doppler examination date, as well as at 6 and 12 months post-examination. $\mathrm{BP}$ was measured by clinical nurses using the conventional auscultatory method or automated device on either arm without distinction. Patients were instructed to sit after a 5-min resting period, and three measurements were taken at least 1 min apart and averaged.

The medical management of BP was identical in both the groups throughout the entire follow-up period. Antihypertensive agents were used to target BP (SBP < $140 \mathrm{mmHg}$; DBP $<90 \mathrm{mmHg}$ ) during regular 1-3 months follow-ups at neurology or cardiology outpatient clinics. The number of classes of antihypertensive drugs taken by the patients at each time point were summated and recorded by drug classes, including alpha-blockers, diuretics, nitrates, calcium channel blockers, angiotensin receptor blockers or angiotensinconverting enzyme inhibitors, and/or beta blockers; combination drugs were recorded as total classes (eg, Hyzaar FC, a combo of losartan and hydrochlorothiazide, as 2). Then, the amount of antihypertensive agents in each class at different points of follow-up (ie, 6 months and 12 months) were compared to the amount in each class at baseline and regarded as increased, reduced, or unchanged.

\section{Post-CAS hypotension}

The study recorded consecutive hourly intervals of SBP values for up to $6 \mathrm{~h}$ post-stenting in patients in the CAS group. Data were assessed using previous reported definitions of post-CAS hypotension, including any decrease in SBP within $6 \mathrm{~h}$ of $\geq 30 \mathrm{mmHg},{ }^{7,8} 40 \mathrm{mmHg},{ }^{9}$ or $40 \%$ compared with the pre-stent value, ${ }^{1}$ and a mean decrease in SBP within $6 \mathrm{~h}$ of $\geq 30 \mathrm{mmHg}, 40 \mathrm{mmHg}$, or $40 \%$ compared with the pre-stent value. All the patients who underwent CAS were grouped into either the "greater SBP-reduction at 1 year" group, which was defined as patients with a SBP decrease of greater than or equal to the total average decrease at 1 year, or the "lesser SBP-reduction at 1 year" group, which was defined as patients with a SBP decrease of lesser than the total group average decrease at 1 year.

\section{Outcome measures}

The primary outcome measures were changes in SBP, DBP, and the number of classes of antihypertensive agents that patients received between baseline and follow-up. Secondary outcome measures included the correlation between postCAS SBP reduction at 1 year and post-CAS SBP reduction within $6 \mathrm{~h}$ using post-CAS hypotension definitions.

\section{Statistical analysis}

The Student's $t$-test was used to compare continuous variables between groups, and the chi-square test was applied to test the 
differences in categorical variables. BP differences within a group during the study period were tested by repeated analysis of variance (ANOVA) measurements. If the assumption of sphericity was violated, adjusted $P$-values were calculated using the Greenhouse-Geisser Epsilon ( $\mathrm{G}-\mathrm{G})$ correction. Univariate linear regressions were performed to test the relationship between SBP changes from baseline to 6 or 12 months of follow-up in both the groups. This relationship was further analyzed using multivariate linear regression and adjusting for potential confounding factors, including diabetic mellitus (DM), smoking, stroke, and changes in the number of classes of antihypertensive agents patients received. In addition, univariate linear regression was used to determine correlations between SBP reductions at 1 year and the maximal or mean SBP reductions $6 \mathrm{~h}$ post-CAS. The statistical significance was set at a $P$-value $<0.05$. Analyses were performed using the SAS statistical software 9.1 (SAS Institute, Cary, NC, USA).

\section{Results}

\section{Baseline characteristics}

A total of 202 patients were recruited for this study (Figure 1). After omitting patients who met the exclusion criteria, 72 patients were enrolled in the CAS group and 82 in the medication group. According to the baseline characteristics (Table 1), the number of patients who smoked was significantly higher in the CAS group than in the medication group (55.7\% vs $33.7 \%$ ). There were also more patients with a history of stroke in the CAS group than in the medication group $(57.1 \%$ vs $41.5 \%$ ). However, more patients in the medication group had DM than in the CAS group (53\% vs $34.3 \%$ ). Other baseline characteristics were similar between groups.

Mean baseline BP was also similar between groups (Table 2). However, the mean baseline SBP was at borderline, which is statistically and significantly higher in the CAS group than in the medication group. The number of classes of antihypertensive agents that patients received at baseline was similar between groups; both the groups used nearly two classes of antihypertensive agents on average at baseline.

\section{Changes of BPs and number of classes of antihypertensive agents used at different time points}

While comparing the SBP and DBP at different time points from baseline to 1 year of follow-up in each group (Table 2), it was found that the CAS group had large decreases in SBP and DBP $6 \mathrm{~h}$ post-stenting (mean SBP, $111.36 \mathrm{mmHg}$; mean DBP, $56.17 \mathrm{mmHg}$ ), which gradually increased 3 days (mean SBP, $121.11 \mathrm{mmHg}$; mean DBP, $65.07 \mathrm{mmHg}$ ) and 1 month (mean SBP, $128.35 \mathrm{mmHg}$; mean DBP, $73.53 \mathrm{mmHg}$ ) poststenting. Mean BP slightly decreased again at 6 months (mean SBP, $128.03 \mathrm{mmHg}$; mean DBP, $72.24 \mathrm{mmHg}$ ) and 12 months post-stenting (mean SBP, $124.93 \mathrm{mmHg}$; mean DBP, $70.08 \mathrm{mmHg}$ ). ANOVA demonstrated that SBP and DBP significantly decreased in the CAS group throughout follow-up (G-G adjusted, $P<0.0001$ and $\mathrm{G}-\mathrm{G}$

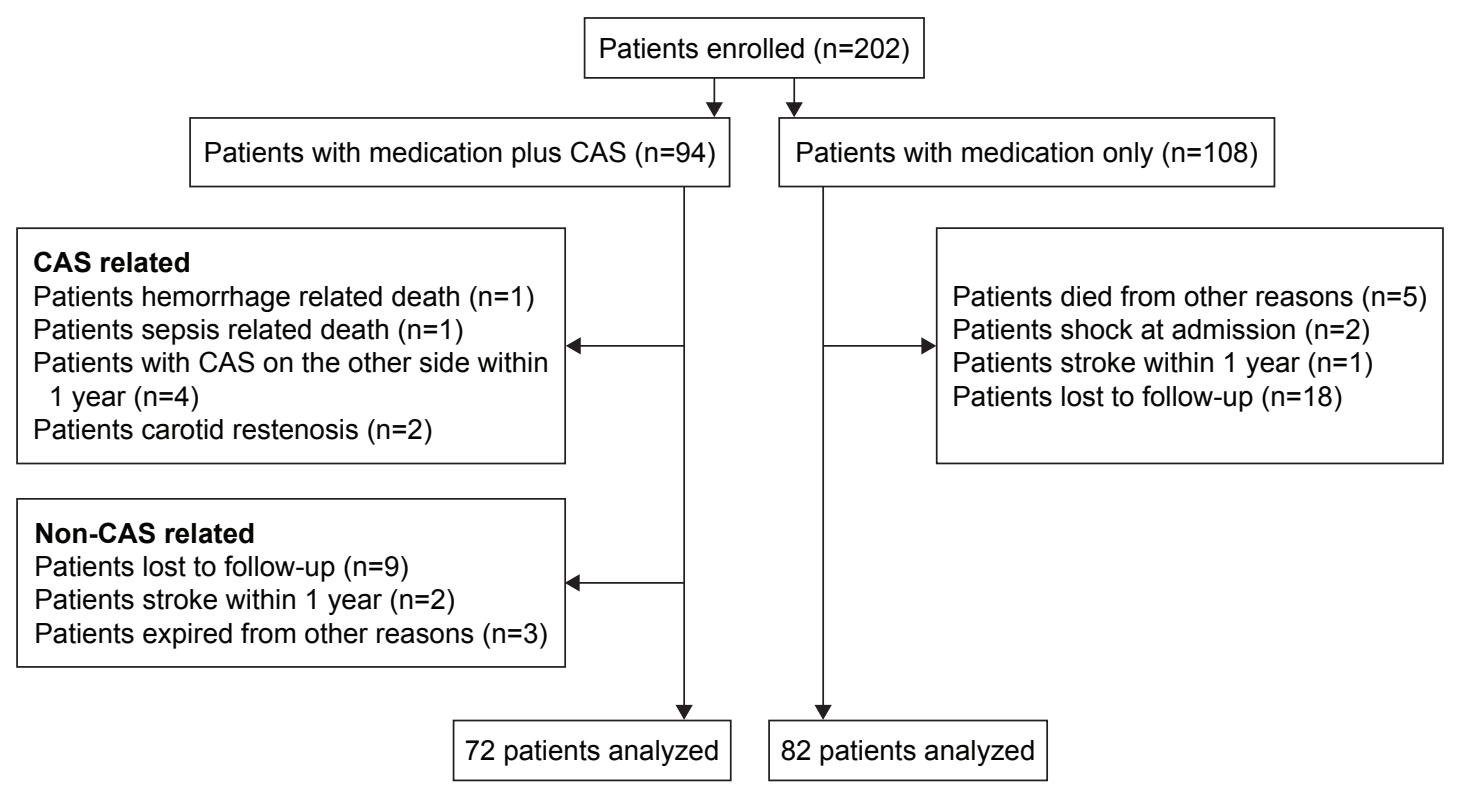

Figure I Flow chart of patients enrolled and reasons for exclusion. Abbreviation: CAS, carotid artery stenting. 
Table I Comparison of baseline characteristics between medication and CAS groups

\begin{tabular}{llll}
\hline & $\begin{array}{l}\text { Medication } \\
(\mathbf{n}=\mathbf{8 2})\end{array}$ & $\begin{array}{l}\text { CAS } \\
(\mathbf{n}=\mathbf{7 2})\end{array}$ & P-value \\
\hline Age (mean \pm SD) & $73(10.2)$ & $70.64(8.9)$ & 0.1291 \\
Sex (male) & $58(69.9)$ & $56(80.0)$ & 0.1266 \\
Medical history & & & \\
$\quad$ Diabetes mellitus & $44(53.0)$ & $24(34.3)$ & 0.0223 \\
Hypertension & $77(92.8)$ & $59(84.3)$ & 0.1097 \\
Treated hyperlipidemia & $43(51.8)$ & $37(52.9)$ & 0.7691 \\
Coronary artery disease & $40(48.2)$ & $43(61.4)$ & 0.0742 \\
Angina & $17(20.5)$ & $10(14.3)$ & 0.2804 \\
Congestive heart failure & $9(10.8)$ & $4(5.71)$ & 0.2362 \\
Myocardial ischemia & $6(7.2)$ & $6(8.6)$ & 0.5765 \\
CABG & $6(7.2)$ & $12(17.1)$ & 0.0674 \\
Atrial fibrillation & $7(8.4)$ & $2(2.9)$ & 0.1332 \\
Peripheral artery & $12(14.5)$ & $4(5.8)$ & 0.0736 \\
$\quad$ occlusive disease & & & \\
Stroke types & & & \\
$\quad$ Transient ischemic attack & $32(38.6)$ & $19(27.1)$ & 0.2191 \\
Stroke & $34(41.5)$ & $40(57.1)$ & 0.0367 \\
Smoking & $28(33.7)$ & $39(55.7)$ & 0.0063 \\
\hline
\end{tabular}

Note: Values presented as number (percentage) unless stated otherwise.

Abbreviations: CABG, coronary artery bypass grafting; CAS, carotid artery stenting.

adjusted, $P<0.0001$, respectively); this was not seen in the medication group ( $\mathrm{G}-\mathrm{G}$ adjusted, $P=0.9180$ and $\mathrm{G}-\mathrm{G}$ adjusted, $P=0.4071$, respectively). Using univariate linear regression model, this study found that patients in the CAS group had a greater decrease in SBP (average, $12.05 \mathrm{mmHg}$ ), compared with those in the medication group after 6 months of follow-up ( $\beta=-12.05, P=0.0005)$. This tendency remained (average, $13.56 \mathrm{mmHg}$ ) after adjusting for DM, smoking, stroke, and changes in the number of classes of antihypertensive agents $(\beta=-13.56, P=0.0002)$. A greater BP-lowering effect was observed at 12 months of follow-up in the CAS group, compared with the medication group. A greater reduction in SBP (average, $16.98 \mathrm{mmHg}$ ) was observed in CAS group ( $\beta=-16.98, P<0.0001)$ after adjusting for confounding factors.

The mean number of classes of antihypertensive agents used were similar between the medication at 6 months (1.82 vs $2.03, P=0.3747)$ and 12 months ( 1.88 vs 1.96 , $P=0.8681$ ) of follow-up (Table 2). Similarly, there were no differences between the groups when the study compared the amount in each class of antihypertensive agents at different points of follow-up (ie, 6 and 12 months) to the baseline (data not shown).

\section{Correlation between post-CAS hypotension and SBP reduction $6 \mathrm{~h}$ and I year post-CAS}

"Greater SBP reduction at 1 year" was defined as patients having greater than or equal to the mean post-CAS SBP reduction at 1 year (which was $15 \mathrm{mmHg}$; Table 3). When defining post-CAS hypotension as "any SBP drop of $\geq 40$ mmHg 6 h post-CAS", significantly more patients had postCAS hypotension in the group of greater SBP reduction at 1 year compared to those in the group of lesser SBP reduction during the same duration ( $80 \%$ vs $46 \%, P=0.0029$ ). Similarly, when defining post-CAS hypotension as "mean SBP drop of $\geq 30 \mathrm{mmHg} 6 \mathrm{~h}$ post-CAS", significantly more patients had post-CAS hypotension in the group of greater SBP reduction at 1 year, compared to those in the group of lesser SBP reduction during the same duration $(57.1 \%$ vs $27 \%, P=0.0096$ ).

A significant positive correlation $(\beta=0.47 \pm 0.10$, $P<0.0001)$ was seen between post-CAS SBP reduction at 1 year and maximal SBP reduction $6 \mathrm{~h}$ post-CAS (Figure $2 \mathrm{~A}$ ). A similar linear relationship ( $\beta=0.20 \pm 0.07, P=0.0067$ ) was observed between SBP reduction 1 year post-CAS and the mean SBP reduction $6 \mathrm{~h}$ post-CAS (Figure 2B).

Table 2 Comparisons between the medication group and the CAS group in terms of changes in SBPs, DBPs, and the number of classes of antihypertensive agents used at different time periods

\begin{tabular}{|c|c|c|c|c|c|c|c|c|c|}
\hline & \multicolumn{3}{|l|}{ SBP } & \multicolumn{3}{|l|}{ DBP } & \multicolumn{3}{|c|}{$\begin{array}{l}\text { Number of classes of } \\
\text { antihypertensive agent }\end{array}$} \\
\hline & Medication & CAS & $P$-value ${ }^{a}$ & Medication & CAS & $P$-value ${ }^{a}$ & Medication & CAS & $P$-value ${ }^{a}$ \\
\hline & Mean & Mean & & Mean & Mean & & Mean & Mean & \\
\hline Baseline & I34.8| (16.8) & 140.04 (16.2) & 0.0508 & $74.16(14.1)$ & $76.29(10.2)$ & 0.2883 & $1.77(1.35)$ & $1.89(1.37)$ & 0.7939 \\
\hline Post-CAS $6 \mathrm{~h}$ & & III.36 (20.7) & & & $56.17(13.8)$ & & & & \\
\hline Post-CAS 3 days & & $121.1 \mid(13.2)$ & & & $65.07(11.1)$ & & & & \\
\hline Post-CAS I month & & $128.35(14.5)$ & & & $73.53(12.3)$ & & & & \\
\hline Post-CAS 6 months & I 34.84 (|8.0) & $128.03(17.9)$ & 0.0199 & $73.88(10.1)$ & 72.24 (II.9) & 0.3535 & $1.82(1.28)$ & $2.03(1.26)$ & 0.3747 \\
\hline Post-CAS I year & 135.59 (20.6) & $124.93(13.8)$ & 0.0003 & $72.33(11.0)$ & $70.08(10.1)$ & 0.1903 & $1.88(1.38)$ & $1.96(1.25)$ & 0.8681 \\
\hline G-G adjusted $P$-value ${ }^{b}$ & 0.9180 & $<0.0001$ & & 0.4071 & $<0.0001$ & & - & - & - \\
\hline
\end{tabular}

Notes: ${ }^{a} P$-value from Student's $t$-test; ${ }^{b} \mathrm{G}-\mathrm{G}$ adjusted $P$-value from repeated measures ANOVA. Values are presented as the mean \pm standard deviation.

Abbreviations: CAS, carotid artery stenting; DBP, diastolic blood pressure; SBP, systolic blood pressure; G-G, Greenhouse-Geisser; ANOVA, analysis of variance. 
Table 3 Correlations between each definitions of post-CAS hypotension within $6 \mathrm{~h}$ and I year post-CAS SBP reductions

\begin{tabular}{|c|c|c|c|c|}
\hline $\begin{array}{l}\text { Definitions of post-CAS } \\
\text { hypotension within } 6 \mathrm{~h}\end{array}$ & Overall $(n=72)$ & $\begin{array}{l}\text { Lesser SBP reduction } \\
\text { at I year }(n=37)\end{array}$ & $\begin{array}{l}\text { Greater SBP reduction } \\
\text { at I year }(n=35)\end{array}$ & $P$-value \\
\hline \multicolumn{5}{|l|}{ Any SBP drop } \\
\hline$\geq 30 \mathrm{mmHg}$ & $55(76.4)$ & $25(67.6)$ & $30(85.7)$ & 0.0700 \\
\hline$\geq 40 \mathrm{mmHg}$ & $45(62.5)$ & $17(46.0)$ & $28(80.0)$ & 0.0029 \\
\hline$\geq 40 \%$ of present SBP & $18(25.0)$ & $6(16.2)$ & $12(34.3)$ & 0.0768 \\
\hline \multicolumn{5}{|l|}{ Mean SBP drop } \\
\hline$\geq 30 \mathrm{mmHg}$ & $30(4 \mid .7)$ & $10(27.0)$ & $20(57.1)$ & 0.0096 \\
\hline$\geq 40 \mathrm{mmHg}$ & II (I5.3) & $3(9.1)$ & $8(22.9)$ & 0.0821 \\
\hline$\geq 40 \%$ of present SBP & $5(6.9)$ & $2(5.4)$ & $3(8.6)$ & 0.6696 \\
\hline
\end{tabular}

Note: Values are presented as the number (percentage) of patients with post-CAS hypotension within $6 \mathrm{~h}$.

Abbreviations: CAS, carotid artery stenting; SBP, systolic blood pressure.

\section{Discussion}

This study demonstrated that patients undergoing CAS for severe carotid artery stenosis had long-term benefits in BP control. Specifically, the research observed a reduction in SBP 1 year post-CAS, despite changes in the number of classes of antihypertensive agent required for optimal BP control. This study holds the first to evaluate the long-term changes in BP levels and the number of classes of antihypertensive agents used between a group of patients who underwent CAS and received medical treatment, and patients who received just medical treatments. Furthermore, this study identified a correlation between BP reductions $6 \mathrm{~h}$ and 1 year post-CAS. Compared with baseline SBP, any SBP decrease of $\geq 40 \mathrm{mmHg}$ or a decrease of $\geq 30 \mathrm{mmHg}$ of mean SBP $6 \mathrm{~h}$ post-CAS may be a predictive factor, indicating a significant decrease in $\mathrm{BP}$ in the long term. It may also be important in adjusting antihypertensive agents during BP monitoring.
Early post-procedural decreases in BP have been observed rather commonly after patients undergo CAS, ${ }^{10-12}$ and several studies have been proposed to confirm whether such condition would persist. ${ }^{2-4,13}$ In 2003, McKevitt et al recruited 49 patients with carotid endarterectomy (CEA) and 55 patients with different types of endovascular treatment and found significant BP reduction solely in CEA groups for up to 6 months post-procedure. ${ }^{13}$ However, it is believed that the results were inconclusive, because there was no record of the number of classes of antihypertensive agents patients received and the author included a limited number of cases in their study. A much larger and multicentered analysis was later conducted with participants (587 patients with CAS and 637 patients with CEA) from the International Carotid Stenting Study. ${ }^{2}$ The investigators showed that BP-lowering effects were significant in 6 months post-CAS or-CEA, although the reductions were seen only in SBPs
A

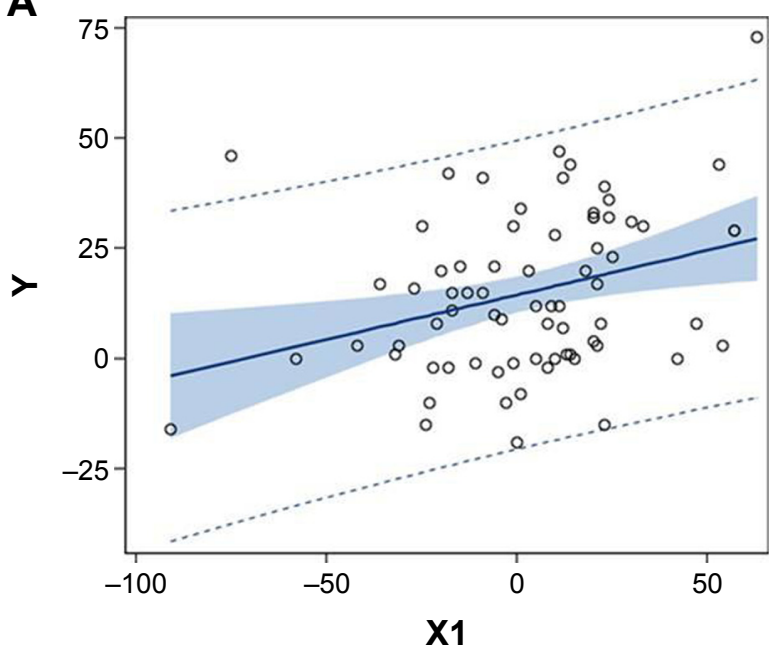

B

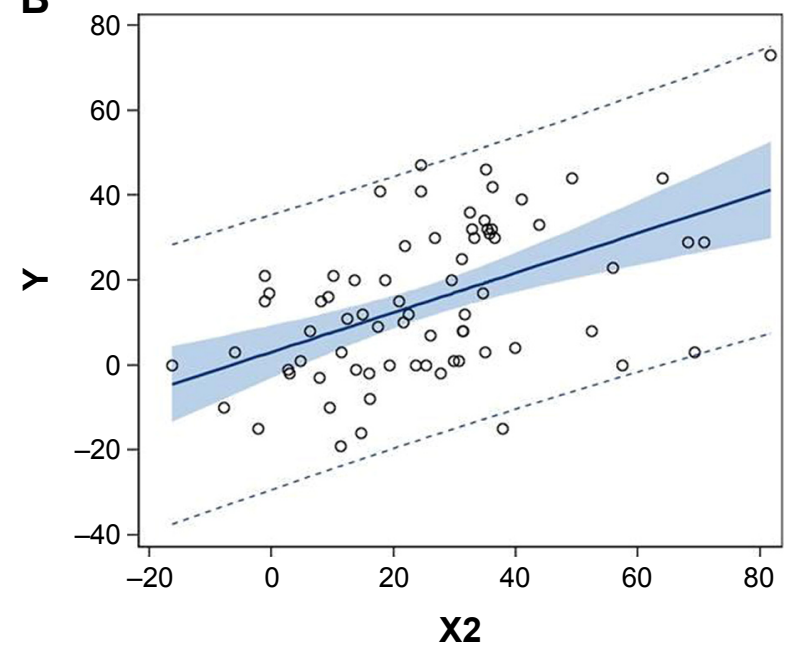

Figure 2 Correlation between SBP reductions I year and $6 \mathrm{~h}$ post-CAS.

Notes: Significant positive correlations were seen between $(\mathbf{A})$ SBP reduction I year post-CAS $(\mathrm{Y})$ and the maximal SBP reduction $6 \mathrm{~h}$ post-CAS (XI); (B) SBP reduction I year post-CAS $(Y)$ and mean SBP reduction $6 \mathrm{~h}$ post-CAS $(X 2)$.

Abbreviations: CAS, carotid artery stenting; SBP, systolic blood pressure. 
and maintained in only patients who underwent CEA after 1 year. Furthermore, after accounting for the additional BPlowering effects that may have been derived from antihypertensive agents, it was concluded that fewer patients were required to use antihypertensive agents post-CAS, compared with post-CEA. These data may explain why there were no statistical significant BP reductions in CAS after 1 year in this study. Furthermore, because no detailed records of the antihypertensive agents used, including their classes and dosage, were collected, the interpretation of the negligible BP-lowering effect from CAS may be rather imprudent. In 2012, Chung et $\mathrm{al}^{3}$ evaluated 45 patients with CEA and 32 patients with CAS and found that patients who underwent CAS had significant long-term BP reductions in 12 months post-procedure, compared with those who underwent CEA. This study further supported the BP-lowering effect of CAS by showing that fewer patients needed additional antihypertensive agents, and more patients were on a lower number of agents required. In 2014, Chung et $\mathrm{al}^{4}$ showed similar BP-lowering effects of CAS analyzed using a different study design that included 107 symptomatic carotid artery stenosis $(>50 \%)$ patients with no change in antihypertensive agents 1 year post-CAS. It should be noted that the present study protocol differs from that study in that this study 1) included asymptomatic patients; 2) evaluated changes in the number of classes of antihypertensive agents that the patients received, which is more common in clinical practice; and 3) carotid stenotic patients without receiving CAS were used as a control group. Thus, we reiterate the additional BP-lowering effect of CAS and the importance of how antihypertensive agents acquired in each study would have difference onto BP changes after CAS.

The second part of the study determined the possible predictive value of patients with significant BP reduction 1 year post-CAS, compared with the BP status $6 \mathrm{~h}$ post-CAS. No study thus far has evaluated the role of possible predictors of decreased BP in the long term by using each definition of short-term post-CAS hypotension. The reasoning for using a 6-h time limit to measure post-CAS hypotension is that a previous analysis demonstrated that a majority $(92 \%, n=23)$ of the first episode of post-CAS hypotension occurred during that time frame post-CAS. ${ }^{1}$ The clinical significance of this phenomenon highlights that patients may be more prone to long-term BP-lowering effects after CAS especially since different types of antihypertensive drugs may have a different impact on adrenergic or sympathetic neural drive. Furthermore, previous experiments showed that diuretics or long-term calcium antagonists may have neutral effects on the adrenergic drive or even trigger a sympathoexcitation. ${ }^{14}$ Alternatively, it should be noted that beta-blockers produce a sympathoinhibition and sympathetic deactivation with drugs acting on the renin-angiotensin system, such as angiotensinconverting enzyme inhibitors or angiotensin II receptor blockers. ${ }^{15}$ Although no such clear correlation was observed in this study - of the 44 patients who used either diuretics or calcium antagonists before CAS, only 17 had significant BP-lowering effect after 1 year - this can be promising in the future in a much larger study.

Previous studies have suggested that mechanical stretch and increased distension of the carotid sinus from the compression of stent dilatation acts as the key reason for $\mathrm{BP}$ decreasing post-CAS. ${ }^{13,16}$ Changes in the baroreflex function in terms of baroreflex sensitivity (BRS) has also been recently suggested. In a study, investigators compared the short-term ( 8 and $24 \mathrm{~h}$ post-procedure) effects on BRS in patients who underwent CEA and CAS procedures and found that the parasympathetic predominance with hypotensive effect was shown only in patients who underwent CAS. ${ }^{17}$ Although the ability of the baroreceptor to buffer acute changes in arterial BP through modulation in sympathetic nerve activity is well established, its role in the long-term role has been debated since the 1970s. ${ }^{18}$ Animal and human studies have demonstrated that prolonged activation of carotid baroreflex may produce significant and sustained BP reductions without any trend for adaptation. ${ }^{19,20}$ Carotid atherosclerosis and stenosis have also been reported to be associated with reduced BRS. ${ }^{21}$ In an another minor study, investigators sought to assess long-term BRS in patients who underwent CAS and hypothesized that CAS could restore the BRS and result in a better long-term control of BP. ${ }^{22}$ Surprisingly, the authors did not observe a significant improvement in BRS from baseline. However, this study believes that the data need to be re-evaluated, because the investigators used a short-term study period (6 months) and has a smaller study population. The possible restoration of BRS after 1-year post-CAS needs to be studied further.

This study had several limitations. Due to the retrospective data collection, the protocol of antihypertensive agent usage in controlling BPs was not standardized by different attending physicians; hence, bias may have produced statistical significance. In addition, the small number of participants included may have yielded unsubstantial conclusions. The study may have also underestimated the occurrences of actual post-CAS hypotension when setting the time period to $6 \mathrm{~h}$, though this specific time setting is considered to be more practical clinically in recording hourly BPs. 


\section{Conclusion}

This study shows that the patients undergoing CAS for severe carotid artery stenosis may benefit from long-term BP control, compared with those who receive only medication, despite changes in the number of classes of antihypertensive agent required for optimal BP control. In addition, this study illustrates the possible predictive value of a significant long-term reduction of $\mathrm{BP}$ if there is any SBP decrease of $\geq 40 \mathrm{mmHg}$ or a decrease of $\geq 30 \mathrm{mmHg}$ of mean SBP $6 \mathrm{~h}$ post-CAS, compared with baseline SBP. This may be an important factor when adjusting antihypertensive agents during BP monitoring.

\section{Acknowledgment}

This work was supported by a grant from Shin Kong Wu Ho-Su Memorial Hospital (SKH-8302-102-DR-15).

\section{Disclosure}

The authors report no conflicts of interest in this work.

\section{References}

1. Lavoie P, Rutledge J, Dawoud MA, Mazumdar M, Riina H, Gobin YP. Predictors and timing of hypotension and bradycardia after carotid artery stenting. AJNR Am J Neuroradiol. 2008;29(10):1942-1947.

2. Altinbas A, Algra A, Brown MM, et al. Effects of carotid endarterectomy or stenting on blood pressure in the International Carotid Stenting Study (ICSS). Stroke. 2011;42(12):3491-3496.

3. Chung J, Kim BM, Paik HK, Hyun DK, Park H. Effects of carotid artery stenosis treatment on blood pressure. J Neurosurg. 2012;117(4): 755-760.

4. Chung J, Kim YB, Hong CK, et al. Blood pressure-lowering effect of carotid artery stenting in patients with symptomatic carotid artery stenosis. Acta Neurochir. 2014;156(1):69-75.

5. Trocciola SM, Chaer RA, Lin SC, et al. Analysis of parameters associated with hypotension requiring vasopressor support after carotid angioplasty and stenting. J Vasc Surg. 2006;43(4):714-720.

6. Hwang CS, Liao KM, Tegeler $\mathrm{CH}$. A multiple regression model of combined duplex criteria for detecting threshold carotid stenosis and predicting the exact degree of carotid stenosis. J Neuroimaging. 2003;13(4): 324-329.

7. Lin PH, Zhou W, Kougias P, El Sayed HF, Barshes NR, Huynh TT. Factors associated with hypotension and bradycardia after carotid angioplasty and stenting. J Vasc Surg. 2007;46(5):846-853.
8. Nonaka T, Oka S, Miyata K, et al. Prediction of prolonged postprocedural hypotension after carotid artery stenting. Neurosurgery. 2005;57(3): $472-427$.

9. Dangas G, Laird JR Jr, Satler LF, et al. Postprocedural hypotension after carotid artery stent placement: predictors and short- and long-term clinical outcomes. Radiology. 2000;215(3):677-683.

10. Park B, Shapiro D, Dahn M, Arici M. Carotid artery angioplasty with stenting and postprocedure hypotension. Am J Surg. 2005;190(5): 691-695.

11. Nano G, Dalainas I, Bianchi P, et al. Ballooning-induced bradycardia during carotid stenting in primary stenosis and restenosis. Neuroradiology. 2006;48(8):533-536.

12. Bussiere M, Lownie SP, Lee D, Gulka I, Leung A, Pelz DM. Hemodynamic instability during carotid artery stenting: the relative contribution of stent deployment versus balloon dilation. JNeurosurg. 2009;110(5): 905-912.

13. McKevitt FM, Sivaguru A, Venables GS, et al. Effect of treatment of carotid artery stenosis on blood pressure: a comparison of hemodynamic disturbances after carotid endarterectomy and endovascular treatment. Stroke. 2003;34(11):2576-2581.

14. Grassi G. Counteracting the sympathetic nervous system in essential hypertension. Curr Opin Nephrol Hypertens. 2004;13(5):513-519.

15. Grassi G, Trevano FQ, Seravalle G, Scopelliti F, Mancia G. Baroreflex function in hypertension: consequences for antihypertensive therapy. Prog Cardiovasc Dis. 2006;48(6):407-415.

16. Mendelsohn FO, Weissman NJ, Lederman RJ, et al. Acute hemodynamic changes during carotid artery stenting. Am J Cardiol. 1998;82(9): 1077-1081.

17. Yakhou L, Constant I, Merle JC, Laude D, Becquemin JP, Duvaldestin P. Noninvasive investigation of autonomic activity after carotid stenting or carotid endarterectomy. J Vasc Surg. 2006;44(3):472-479.

18. Wustmann K, Kucera JP, Scheffers I, et al. Effects of chronic baroreceptor stimulation on the autonomic cardiovascular regulation in patients with drug-resistant arterial hypertension. Hypertension. 2009; 54(3):530-536.

19. Thrasher TN. Baroreceptors and the long-term control of blood pressure. Exp Physiol. 2004;89(4):331-355.

20. Kroon A, Schmidli J, Scheffers I, et al. Sustained blood pressure reduction by baroreflex activation therapy with a chronically implanted system: 4-year data of Rheos Debut-Ht study in patients with resistant hypertension: 9D.01. J Hypertens. 2010;28:e441.

21. Chao AC, Chern CM, Kuo TB, et al. Noninvasive assessment of spontaneous baroreflex sensitivity and heart rate variability in patients with carotid stenosis. Cerebrovasc Dis. 2003;16(2):151-157.

22. Huang CC, Wu YS, Chen T, et al. Long-term effects of baroreflex function after stenting in patients with carotid artery stenosis. Auton Neurosci. 2010;158(1-2):100-104.
Clinical Interventions in Aging

\section{Publish your work in this journal}

Clinical Interventions in Aging is an international, peer-reviewed journal focusing on evidence-based reports on the value or lack thereof of treatments intended to prevent or delay the onset of maladaptive correlates of aging in human beings. This journal is indexed on PubMed Central, MedLine,

\section{Dovepress}

CAS, Scopus and the Elsevier Bibliographic databases. The manuscript management system is completely online and includes a very quick and fair peer-review system, which is all easy to use. Visit http://www.dovepress. com/testimonials.php to read real quotes from published authors. 